\title{
Excessively High Urinary Beta 2-Microglobulin Level: A Sign of Pathology or Laboratory Error?
}

\author{
Gulsum Feyza Altas ${ }^{1}$, Pinar Akan ${ }^{1}$ and Erkan Cagliyan ${ }^{2}$ \\ 1. Department of Medical Biochemistry, Faculty of Medicine, Dokuz Eylul University, Izmir 35000, Turkey \\ 2. Department of Obstetrics and Gynecology, Faculty of Medicine, Dokuz Eylul University, Izmir 35000, Turkey
}

\begin{abstract}
Measurement of urinary $\beta 2 \mathrm{M}$ (beta 2-microglobulin) is a sensitive and reliable assay for detecting tubular injury, renal toxicity, lymphomas, leukemia, or myeloma. Some chemical substrates may increase the level of $\beta 2 \mathrm{M}$ in-vivo. Elevated $\beta 2 \mathrm{M}$ level in urine is unusual because it rapidly degrades when $\mathrm{pH}$ is below 6 . The level of $\beta 2 \mathrm{M}$ in the bladder can also be used as a marker to assess renal tubular maturation in neonates. $\beta 2 \mathrm{M}$ in the bladder could be a result of fetal megacystis, which is an abnormally enlarged bladder appearing after 10 weeks of gestation, when the fetus begins to produce urine. Identification of the pregnant women instead of the fetus is a common pre-analytical error with samples sent from the gynecology clinic to the laboratory. Here we present the case of a 24-year-old pregnant woman whose urine analysis results indicated excessively high $\beta 2 \mathrm{M}$ level in the urine. The present study could improve the understanding of urinary $\beta 2 \mathrm{M}$ analysis, laboratory errors, and the interpretation of test results.
\end{abstract}

Key words: Pre-analytical error, urinary $\beta 2 \mathrm{M}$, fetal megacystis.

\section{Introduction}

$\beta 2 \mathrm{M}$ (beta 2-microglobulin) is a small molecular weight protein found on the cell surface. It is present in most body fluids (blood, urine, cerebrospinal fluid) and its level rises with conditions that increase cell production and/or destruction or that activates the immune system [1]. Increased serum levels may indicate lymphomas, leukemia, or myeloma; glomerular renal disease; renal transplant rejection; viral infections, especially HIV and cytomegalovirus; or chronic inflammatory processes.

Although the small size of $\beta 2 \mathrm{M}$ allows it to be filtered through the glomerular membrane, typically less than $1 \%$ of the filtered $\beta 2 \mathrm{M}$ is excreted in the urine [2]. The majority of $\beta 2 \mathrm{M}$ in the filtrate is reabsorbed and catabolized by renal proximal tubular cells [3]. Therefore, $\beta 2 \mathrm{M}$ serves as a useful biomarker to evaluate both glomerular and tubular function [4].

Measurements of proteinuria and enzyme-uria are

Corresponding author: Gulsum Feyza Altas, M.D., research assistant, research fields: clinical biochemistry and laboratory diagnostics. simple, convenient and non-invasive diagnosis methods used for the early detection of renal damage $[5,6]$. Measurement of urinary $\beta 2 \mathrm{M}$ is one of the most sensitive and reliable assay for detecting tubular injury [7]. Increased levels in the urine indicate renal tubule disease; drug-induced renal toxicity; heavy metal-induced renal disease; lymphomas, leukemia, or myeloma; or AIDS. Toxic elements such as cadmium and mercury, which cause tubular damage, significantly increase the amount of $\beta 2 \mathrm{M}$ in the urine. In occupational medicine, the determination of $\beta 2 \mathrm{M}$ in urine is regarded as the most sensitive test for early detection of tubular damage from excessive exposure to cadmium [8]. Drugs that can cause tubular dysfunction through toxicity, primarily aminoglycosides and lithium, cause an increase in the amount of $\beta 2 \mathrm{M}$ that is in eliminated in the urine. The nephrotoxicity of water-soluble urographic contrast material is also well recognized. Fulvio Stacul et al. demonstrated that contrast material causes significant increase in the urinary excretion of $\beta 2 \mathrm{M}$.

$\beta 2 \mathrm{M}$ can be used as a marker to assess renal tubular maturation in neonates. Either 24-hour urine or random 
urine samples can be used. In the fetus, serum creatinine cannot be used as a marker of renal function because it crosses the placenta and is cleared by the mother. This is not the case for $\beta 2 \mathrm{M}$, which has been proposed as a marker for fetal renal function [9]. However, data on the prediction of postnatal morbidity based on fetal serum $\beta 2 \mathrm{M}$ levels remain scarce. Indeed, the number of fetuses investigated to date is small and the study population heterogeneous, comprising both unilateral and bilateral uropathies and nephropathies [10]. Fetal serum $\beta 2 \mathrm{M}$ is a marker for renal function and predicts postnatal serum creatinine in bilateral or low fetal obstructive uropathy [10]. Studies have demonstrated higher urinary $\beta 2 \mathrm{M}$ concentrations in children with ureteropelvic junction obstruction compared with healthy controls $[11,12]$.

\section{Case Presentation}

In October 2016, a random urine specimen with request for $\beta 2 \mathrm{M}$ analysis was sent from the gynecology and obstetrics clinic to the central laboratory of the university hospital. According to the patient identification number in the hospital information system, the sample belonged to a 24-year-old (gravida 1 , para 0 ) healthy pregnant woman at 21 weeks and 2 days gestation. There was no additional information about the patient.

In the central laboratory, $\beta 2 \mathrm{M}$ level in the urine specimen was analyzed by turbidimetric method (Binding site, UK) and was determined to be excessively high $(\beta 2 \mathrm{M}$ : $17.3 \mathrm{mg} / \mathrm{L}$, creatinine: 1,572 $\mathrm{mg} / \mathrm{g})$. The analysis was repeated at least three times with and without dilution. Calibration and quality control values of the analytic method were optimal. When a laboratory specialist contacted the physician to report the $\beta 2 \mathrm{M}$ result, it was learned that the urine specimen was obtained from the bladder of a single male fetus presenting with oligohydramnios.

On sonographic evaluation at 18 weeks gestation, placenta anterior was observed, BPD (biparietal diameter) was measured as 18 weeks 4 days, HC (head circumference) was also determined as 18 weeks 5 days, and FL (femur length) was measured as 17 weeks 6 days; AC (abdominal circumference) could not be assessed. Anhydramnios was diagnosed based on findings of a very low AFI (amniotic fluid index) $(<4$ $\mathrm{cm})$. The fetal heart rate was normal. After clinical evaluation, bladder shunt therapy was performed in order to preserve amniotic fluid volume and to protect the lungs rather than the kidneys.

In November 2016, anhydramnios persisted, and the patient was evaluated for amnioinfusion and repeated shunt application at 26 weeks gestation. The patient did not accept the perinatology council's recommendation to terminate the pregnancy. On December 21st, the fetus was born with renal failure, patent ductus arteriosus, cryptorchidism, talipes equinovarus, and bilateral renal hypoplasia.

In this case, the high level of urine $\beta 2 \mathrm{M}$ was a result of fetal megacystis.

\section{Discussion}

$\beta 2 \mathrm{M}$ levels are generally assayed by immunoassay methods such as radial immunodiffusion, radioimmunoassay, enzyme immunoassay, fluoroimmunoassay, and latex immunoassay [13], but the analytical performance of these techniques varies. Single radial immunodiffusion was initially used for determination of $\beta 2 \mathrm{M}$ in human urine or serum, but this method is not sensitive enough to detect $\beta 2 \mathrm{M}$ in reference population urine without a preliminary concentration step. RIA (radioimmunoassay) methods were then developed which can accurately measure $\beta 2 \mathrm{M}$ in normal urine or serum without sample preconcentration [1]. In the past 30 years, several techniques such as nephelometry, turbidimetry, and particle counting have been developed as quantitative assays based on latex agglutination [8, 14-16]. It has been shown that the latex immunoassay method has higher specificity, sensitivity, and precision than the other assays. In addition, the latex immunoassay protocol is simpler [8]. In contrast, there are many 
disadvantages associated with the use of radioisotopes (shelf life, health hazards, etc.) in RIA assessment.

In our study, we analysed urinary $\beta 2 \mathrm{M}$ levels by immunoturbidimetric method based on latex immunoassay (Binding site, UK). The reference interval was $<0.2 \mathrm{mg} / \mathrm{L}$ for urinary $\beta 2 \mathrm{M}$. The reportable range of our method has been declared by the manufacturer as between 0.6 and $20 \mathrm{mg} / \mathrm{L} \mathrm{[17].}$ $\beta 2 \mathrm{M}$ value of the patient specimen was obtained with $1 / 10$ dilution $(17.3 \mathrm{mg} / \mathrm{L})$. The within-run precision was $0.71 \% \mathrm{CV}$ (coefficient of variation) and total precision was $4.24 \% \mathrm{CV}$. TE (total error) was calculated as $13.5 \%$ and was below the acceptable total error of the $\beta 2 \mathrm{M}(\mathrm{TEa} ; 30 \%)$ [18].

In common opinion, the immunoturbidimetric method described is rapid, easy to perform, and utilizes small quantities of reagent, sample, and antiserum, making it cheaper than commercial kit methods. The method has been found to be very reliable in practice, and although much less sensitive than enzyme-linked and radioimmunoassay, it covers the range of $\beta 2 \mathrm{M}$ values in serum and urine that are relevant to the use of this assay for the assessment of prognosis in patients with myelomatosis [13]. Although analytical performance of our method was generally adequate to determine $\beta 2 \mathrm{M}$, it could be better if the result was obtained without any dilution of the specimen to reduce possible analytical errors and unnecessary test repeating.

$\beta 2$-microglobulin testing is used to monitor fetal megacystis. Fetal megacystis is an abnormally enlarged bladder (longitudinal diameter is $6-8 \mathrm{~mm}$ or bladder/CRL ratio is $\geq 13 \%$ ) appearing after 10 weeks of gestational age, when the fetus starts producing urine. Increases in the dimensions of the fetal bladder after 10 weeks of gestation are detected by routine ultrasound [19]. It has been suggested that biochemical analysis of fetal urine could provide valuable insights into prognosis for renal function, but there is no consensus regarding the best test or combination of tests. While good correlation has been observed between fetal serum $\beta 2 \mathrm{M}$ and postnatal serum creatinine after 24 weeks, fetal blood sampling is difficult to perform before 20 weeks [20,21].

In the presented case, the $\beta 2 \mathrm{M}$ level $(17.3 \mathrm{mg} / \mathrm{L})$ of the fetus indicated poor prognosis (cut-off value $<2$ $\mathrm{mg} / \mathrm{L}$ ) [22]. The use of $\beta 2 \mathrm{M}$ as a marker of developing renal damage in utero may prove useful in the future for indicating the need to intervene to prevent not only pulmonary hypoplasia but also long-term renal damage [23]. For fetal megacystis, other adverse prognostic factors for fetal treatment outcomes are the presence of renal dysplasia, changes in serum electrolytes (calcium and sodium), and severe oligohydramnios beginning in the first trimester. In a study by Crombleholme et al. fetuses with declining or decreased amniotic fluid volumes and urinary electrolyte levels were associated with a good prognosis [21, 24, 25].

Freedman et al. reported the long-term outcomes in children after vesico amniotic shunt placement: nearly $40 \%$ died, and half of the deaths were secondary to pulmonary hypoplasia [26]. Among the survivors, almost $40 \%$ had serious renal impairment. Thus, although vesico amniotic shunting may improve survival in some cases, long-term renal insufficiency, kidney transplantation, or both, as well as pulmonary hypoplasia remain important risks [23].

To decrease laboratory errors for urinary $\beta 2 \mathrm{M}$ analysis, 24-h urine is recommended, but random urine samples can also be used. If using random sampling, the patient should empty the bladder and drink at least $0.5 \mathrm{~L}$ of water. The urine sample should be collected within 1 hour. Samples not processed immediately will grow bacteria that produce urease, which metabolizes urea in the sample to $\mathrm{CO}_{2}$ and $\mathrm{NH}_{3}$ (ammonia), which binds $\mathrm{H}^{+}$to form $\mathrm{NH}_{4}{ }^{+}$, thus increasing the $\mathrm{pH}$ of the sample [27]. This alkaline environment leads to degradation [2]. The $\mathrm{pH}$ of the urine sample should be adjusted to 6-8 using $1 \mathrm{M} \mathrm{HCl}$ if above 8 and 5\% $\mathrm{NaOH}$ if below 6 to avoid degradation [28]. In contrast, the $\mathrm{pH}$ of urine obtained from a fetus in utero is at the desired level. The urine specimen should be 
refrigerated until analysis to prevent significant bacterial growth [27]. Samples may be stored refrigerated at $2-8{ }^{\circ} \mathrm{C}$ for 5 days. For longer storage (up to 6 months), samples should be stored frozen at $-20^{\circ} \mathrm{C}$. To avoid repeated thawing and freezing, the samples should be aliquoted. Bilirubin and hemolysis do not significantly affect the procedure. However, gross lipemia can interfere with results. Urinary $\beta 2 \mathrm{M}$ levels are increased by aminoglicosid antibiotics, carboplatin, cisplatin, cyclosporine, and radiographic contrast media [28].

In our case, the mother had no conditions which would cause an increase in urinary $\beta 2 \mathrm{M}$ level. The actual reason was fetal megacystis, and the urine belonging to the fetus was erroneously sent to the laboratory with the mother's information.

Wrong or missing identification is one of the most frequently reported types of pre-analytical error [29]. Although fetal megacystis occurs in approximately 1 in 1,831 pregnancies, identification of the pregnant women instead of the fetus is a common problem for samples sent from gynecology clinics [19]. The international guideline defines the pre-analytical phase as "steps starting in chronological order, from the clinician's request and including the examination requisition, preparation of the patient, collection of the primary sample, and transportation to and within the laboratory, and ending when the analytical examination procedure begins" [30]. When we take into consider this, there will not be unnecessary loss of test, loss of time and sample rejection. In past years, the parameters about preanalytical phase have been defined as innovative quality indicators such as the ratio of the wrong patient or sample identification. The identification of reliable quality indicators is a crucial step in enabling users to quantify the quality of laboratory services [31]. In our laboratory, the ratio of sample rejection has been used as a quality indicator to monitor the laboratory service. The ratio of the wrong identification can also be regularly used as an indicator to reduce unnecessary expenditures and to increase patient safety. On the other hand, especially for the gynecology clinics, it can be emphasized how important the detailed sample identification is important to the laboratory.

\section{Conclusions}

Our case report shows that the patient identification is a critical to correctly interpret laboratory results and avoid unnecessary repetition of tests. The present study could improve the understanding of urinary $\beta 2 \mathrm{M}$ analysis, pre-analytical laboratory errors and the importance of sample identification.

\section{References}

[1] Forman, D. T. 1982. "Beta-2 Microglobulin. An Immunogenetic Marker of Inflammatory and Malignant Origin." Ann Clin Lab Sci. 12 (6): 447-52.

[2] Burtis, C. A., Ashwood, E. R., and Bruns, D. E. 2012. Tietz Textbook of Clinical Chemistry and Molecular Diagnostics. Elsevier Health Sciences, 2259 p.

[3] Engl, N. 1976. "Serum $\beta 2$-Microglobulin and Glomerular Function.” J. Med. Massachusetts Medical Society 294 (11): 611.

[4] Trof, R. J., Maggio, F., Di Leemreis, J., and Groeneveld, A. B. J. 2006. "Biomarkers Of Acute Renal Injury and Renal Failure." Shock 26 (3): 245-53.

[5] Agents, C. C. 1987. "Contrast Agent Nephrotoxicity." December: 1287-9.

[6] Efstratiadis, G., Pateinakis, P., Tambakoudis, G., Pantzaki, A., Economidou, D., and Memmos, D. 2008. "Contrast Media-Induced Nephropathy: Case Report and Review of the Literature Focusing on Pathogenesis." Hippokratia 12 (2): 87-93.

[7] Zeng, X., Hossain, D., Bostwick, D., Herrera, G., Ballester, B., and Zhang, P. 2014. "Urinary $\beta 2-$ Microglobulin Is A Sensitive Indicator for Renal Tubular Injury.” Sch Arena J. Case Rep. 1 (1): 103-8.

[8] Bernard, A. M. 1981. "Determination of f32-Microglobulin in Human Urine and Serum by Latex Immunoassay." CLIN CHEM Clin Chem. 276 (6): 832-7.

[9] Berry, S. M., Lecolier, B., Smith, R. S., Bercau, G., Dombrowski, M. P., Puder, K. S., et al. 1995. "Predictive Value of Fetal Serum Beta 2-Microglobulin for Neonatal Renal Function.” Lancet (London, England). 345 (8960): 1277-8.

[10] Dommergues, M., Muller, F., Ngo, S., Hohlfeld, P., Oury, J. F., Bidat, L., et al. 2000. "Fetal Serum 32 -Microglobulin Predicts Postnatal Renal Function in Bilateral Uropathies." Kidney Int. 58 (1): 312-6. 
[11] Bartoli, F., Penza, R., Aceto, G., Niglio, F., D’Addato, O., Pastore, V., et al. 2011. "Urinary Epidermal Growth Factor, Monocyte Chemotactic Protein-1, and 32-Microglobulin in Children with Ureteropelvic Junction Obstruction." J. Pediatr Surg. 46 (3): 530-6.

[12] Madsen, M. G., Nørregaard, R., Palmfeldt, J., Olsen, L. H., Frøkiær, J., and Jørgensen, T. M. 2013. "Epidermal Growth Factor and Monocyte Chemotactic Peptide-1: Potential Biomarkers of Urinary Tract Obstruction in Children with Hydronephrosis." J. Pediatr Urol. 9 (6 Pt A): 838-45.

[13] Tillyer, C. R., and Rawal, Y. 1988. "An Immunoturbidimetric Method for the Measurement of Beta-2 Microglobulin in Serum and Plasma on a Centrifugal Analyser.” Ann. Clin. Biochem. 67-72.

[14] Deželić, G., Deželić, N., Muić, N., and Pende, B. 1971. "Latex Particle Agglutination in the Immunochemical System Human Serum Albumin-Anti-human Serum Albumin Rabbit Serum.” Eur. J. Biochem. 20 (4): 553-60.

[15] Cambiaso, C. L., Leek, A. E., De Steenwinkel, F., Billen, J., and Masson, P. L. 1977. "Particle Counting Immunoassay (PACIA). I. A General Method for the Determination of Antibodies, Antigens, and Haptens." J. Immunol Methods 18 (1-2): 33-44.

[16] Grange, J., Roch, A. M., and Quash, G. A. 1977. "Nephelometric Assay of Antigens and Antibodies with Latex Particles.” J. Immunol Methods 18 (3-4): 365-75.

[17] Use, I. 2015. "Human Beta-2 Microglobulin Kit for use on the SPA PLUS ${ }^{\circledR}$ for in vitro Diagnostic Use Only Product Code: LK043." S. May:27-9.

[18] Allowable Total Error Table|Data Innovations [Internet]. [cited 2017 Nov 6]. Available from: https://datainnovations.com/allowable-total-error-table?fi eld_category_type_tid $=$ All\&title $=$ Activated + Clotting $+\mathrm{Ti}$ me.

[19] "Isolated Fetal Megacystis: A Case Report-Gazzetta Medica Italiana Archivio per le Scienze Mediche.” 2014. Minerva Medica Journals 173 (4): 223-8.

[20] Abdennadher, W., Chalouhi, G., Dreux, S., Rosenblatt, J., Favre, R., Guimiot, F., et al. 2015. "Fetal Urine Biochemistry at 13-23 Weeks of Gestation in Lower
Urinary Tract Obstruction: Criteria for In-utero Treatment.” Ultrasound Obstet Gynecol 46 (3): 306-11.

[21] Morris, R. K., Quinlan-Jones, E., Kilby, M. D., and Khan, K. S. 2007. Systematic Review of Accuracy of Fetal Urine Analysis to Predict Poor Postnatal Renal Function in Cases of Congenital Urinary Tract Obstruction. Prenat Diagn. John Wiley \& Sons. 27 (10): 900-11.

[22] "Fetal Serum $\beta 2$-microglobulin Predicts Postnatal Renal Function in Bilateral Uropathies." 2000. Kidney Int. 58 (1): 312-6.

[23] Pinette, M. G., Blackstone, J., Wax, J. R., and Cartin, A. 2003. "Enlarged Fetal Bladder: Differential Diagnosis and Outcomes." J. Clin Ultrasound 31 (6): 328-34.

[24] Clark, T. J., Martin, W. L., Divakaran, T. G., Whittle, M. J., Kilby, M. D., and Khan, K. S. 2003. "Prenatal Bladder Drainage in the Management of Fetal Lower Urinary Tract Obstruction: A Systematic Review and Meta-analysis." Obstet Gynecol. Centre for Reviews and Dissemination (UK) 102 (2): 367-82.

[25] Crombleholme, T. M., Harrison, M. R., Golbus, M. S., et al. 1990. "Fetal Intervention in Obstructive Uropathy: Prognosticindicators and Efficacy of Intervention.” Am. J. Obstet Gynecol. Mosby 162 (5): 1239-44.

[26] Freedman, A. L., Johnson, M. P., Smith, C. A., Gonzalez, R., and Evans, M. I. 1999. "Long-Term Outcome in Children after Antenatal Intervention for Obstructive Uropathies." Lancet 354 (9176): 374-7.

[27] Illustrated AN, Text C. Clinical Biochemistry.

[28] Wu, A. H. B. 2006. Tietz clinical Guide to Laboratory Tests. Saunders/Elsevier, 1798 p.

[29] Plebani, M. 2012. "Quality Indicators to Detect Pre-analytical Errors in Laboratory Testing." Clin. Biochem Rev. The Australian Association of Clinical Biochemists 33 (3): 85-8.

[30] ISO 15189: 2012(en), Medical laboratories - Requirements for Quality and Competence. 2012.

[31] Plebani, M., Sciacovelli, L., Aita, A., Padoan, A., and Chiozza, M. L. 2014. "Quality Indicators to Detect Pre-analytical Errors in Laboratory Testing." Clin. Chim. Acta. 432: 44-8. 